Cuadernos de Trabajo Social

ISSN: 0214-0314

http://dx.doi.org/10.5209/CUTS.56352

\title{
Hacia un nuevo Trabajo Social crítico: el gobierno de lo social en la era neoliberal. Presentación del monogáfico
}

\author{
[en] Toward a new critical Social Work: social governance in the neoliberal era. \\ Presentation of Monograph
}

Sergio García García; César Rendueles Menéndez de Llano

La crisis social que se abrió en España con el pinchazo de la burbuja inmobiliaria, en 2008, y su gestión pública en forma de austeridad han vuelto a colocar en la agenda mediática, académica y política los conflictos relacionados con la exclusión y la desigualdad. En el inicio de la crisis se generalizó una visibilización humanitarista y pacificadora de la pobreza. Los medios de comunicación «espectacularizaron» el malestar, centrando su atención en las acciones filantrópicas de los famosos o exhibiendo en los platós de televisión a los pobres implorando ayuda. El clima de politización que generó el 15-M agrietó esa comprensión consensual de las dinámicas de estratificación que, en cambio, empezaron a entenderse en términos de polarización, conectando los datos y las imágenes del sufrimiento social con los beneficios de las élites empresariales y políticas.

En este ambiente de antagonismo, muchos trabajadores sociales cuestionaron el papel que se les había atribuido como muro de contención del malestar social y experimentaron un proceso de implicación que les llevó a prestar atención a los discursos de las plazas, así como a incluir las prácticas de apoyo mutuo de los movimientos sociales de los barrios en sus «guías de recursos». En algunos casos la dinámica crítica llegó a cristalizar en iniciativas colectivas, como la Marea Naranja o el Foro ServSocial. Se trata de un ciclo de movilización modesto, pero prácticamente inédito en el seno de la intervención social española de las últimas décadas. Como mínimo, ha servido para que una gran cantidad de trabajadores sociales consideren ineludibles los debates en torno al carácter estructural de los problemas sociales y se planteen la necesidad de elaborar alternativas a las recetas profesionales heredadas.

Sin embargo, la reflexividad y la movilización de los trabajadores sociales, tanto en la calle como en las redes sociales, no parece haber dado lugar a una reflexión parsimoniosa sobre cuestiones cruciales relacionadas con el papel que desempeña la intervención en la reproducción social. Se ha popularizado una retórica crítica con la crisis y la austeridad que considera los servicios sociales y el Trabajo Social como aliados leales de las víctimas de la exclusión y la desigualdad. El corolario de esta perspectiva conciliadora es que el objetivo debería ser recuperar los servicios sociales y las estrategias de intervención previas a la crisis, a lo sumo mejorándolos con importantes inyecciones presupuestarias y algunas reformas en materia de derechos sociales y participación de los afectados. En cambio, apenas se han llegado a desarrollar enfoques que cuestionen las propias relaciones de poder ligadas al rol exper- 
to, las metodologías individualistas y las funciones de control del Trabajo Social en el sostenimiento de la explotación laboral y la segregación socioespacial. En todo caso, esas perspectivas críticas han procedido de algunos movimientos sociales o de otras disciplinas, como la sociología y la antropología. Por ese motivo a menudo se han vivido como una amenaza al frágil estatus profesional e institucional logrado por los trabajadores sociales en las últimas décadas y han sido respondidas con un fuerte cierre de filas corporativista e identitario, pero carente de profundidad teórica.

En este contexto, resulta urgente abrir una veta de reflexiones en torno a la contribución del Trabajo Social a la reproducción del orden social, una crítica libre de autocomplacencias y centrada en la utilidad social más que en la identidad profesional. Volver a considerar el Trabajo Social como un medio y no como un fin es el primer paso para recuperar el compromiso de esta disciplina con la justicia social. Para ello, se hace preciso, en primer lugar, desencantar la profesión, asumir que no posee una esencia que la haga per se transformadora, y centrarse en el análisis de los contextos, los usos, los significados y los efectos de las intervenciones concretas. En segundo lugar, es necesario reflexionar sobre los «paraqués» y los posicionamientos - adaptativos, educativos o transformadores (Hernández, 2012) — que se asumen ante las situaciones de intervención.

Este monográfico está dedicado al Trabajo Social como modo de gobierno de lo social en contextos de desigualdad. En primer lugar, hay que señalar que esta propuesta analítica no conlleva una comprensión del Trabajo Social «exclusivamente» en términos de control social; es decir, un enfoque reactivo frente a la descripción del Trabajo Social como modo de ayuda. La dicotomía ayuda versus control oscurece la complejidad de la práctica, las tensiones que la atraviesan y la ambivalencia de sus productos. Las intervenciones sociales producirán efectos diversos y a veces contradictorios en función de la temporalidad (el efecto inmediato de una intervención en un barrio puede ser el reconoci- miento de la diversidad cultural, pero también un posterior reflujo xenófobo), de la composición social de los contextos concretos (dependiendo de la correlación de fuerzas entre actores institucionales, profesionales y vecinales, la misma intervención ampliará la democracia local o no), de los ejes de poder sobre los que se ponga el foco (la acción compensadora sobre una desigualdad de género puede estar ahondando en una desigualdad étnica, como puede ser el empoderamiento de mujeres de minorías sobre postulados eurocéntricos) o de la escala a la que prestemos atención (lo que en un nivel comunitario puede suponer el desarrollo a través de la capacitación, en un nivel estructural puede abrir la puerta a la colonización de la vida local por parte de las relaciones de mercado).

En segundo lugar, este monográfico se basa en una concepción amplia del Trabajo Social que toma en consideración tanto las políticas sociales y los servicios sociales como las prácticas profesionales de intervención social (con independencia de que las pongan en marcha o no titulados en Trabajo Social). La sinécdoque puede parecer injusta, pero esta estrategia facilita el reconocimiento de la maternidad del Trabajo Social sobre el campo de la intervención social para conectar así las críticas contemporáneas con las que ha recibido desde su surgimiento en el siglo XIX.

En tercer lugar, es importante precisar que cuando hablamos de «gobierno» nos referimos al modo de conducir las conductas de los sujetos (Foucault, 2007) y no a las instituciones de gobierno. Entender el gobierno como actividad y no sólo como organización institucional (Laval y Dardot, 2013), permite superar la perspectiva estadocéntrica para reparar en las prácticas que una multiplicidad de agentes implicados en lo social llevan a cabo en la conformación de un orden social determinado (desde los responsables políticos a los propios usuarios de los dispositivos de atención social, pasando por los expertos, los medios de comunicación y la sociedad civil).

En cuarto lugar, entendemos lo social como un campo de preocupaciones y actuaciones reformistas sobre los sectores menos 
favorecidos por el modo de producción capitalista, una «invención» necesaria para volver gobernable una sociedad que había optado contradictoriamente por un régimen político democrático y por un sistema económico desigualitario (Donzelot, 2007).

$\mathrm{Y}$ en quinto y último lugar, apuntamos a los contextos de desigualdad, tratando de situarnos en el capitalismo en su fase neoliberal, aludiendo fundamentalmente a sus manifestaciones de clase, pero sin olvidar su estrecha conexión con las discriminaciones de género, las segregaciones racialistas y otros ejes contemporáneos de estructuración jerárquica del cuerpo social.

Antes de presentar los contenidos del monográfico, estimamos necesario responder a algunas preguntas que nos situarán en las puertas del problema que queremos abordar (los usos del Trabajo Social en el neoliberalismo como un dispositivo de control o reproductivo del orden): ¿Qué supuso la extensión histórica de las relaciones de mercado y qué condiciones hicieron posible la emergencia de lo social? ¿Cuál ha sido históricamente el papel específico del Trabajo Social en el gobierno de lo social según las críticas formuladas desde dentro y desde fuera de la profesión? ¿Qué implicaciones materiales e ideológicas tiene el neoliberalismo? ¿Qué modos de instrumentalización y cooptación de discursos, otrora críticos, del Trabajo Social sirven hoy para gobernar la desigualdad?

\section{La formación del capitalismo y la inven- ción de lo social}

Al menos desde el siglo XVIII, el ideal del mercado libre autorregulado ocupa un lugar central entre los mecanismos de construcción de hegemonía de la sociedad moderna. A través de una amplia constelación intelectual se refinó la idea de que, en una economía compleja, el sistema de precios es el medio idóneo para alcanzar una asignación óptima de los recursos (Perelman, 2000). Desde esta perspectiva, los precios transmiten a un coste mínimo información fragmentaria sobre las preferencias individuales que se agrega automáticamente, de manera que se genera un nivel de coordinación social mayor que el que ninguna institución organizadora podría alcanzar: la intervención centralizada no hace más que distorsionar el flujo de información impidiendo la coordinación óptima. Desde el primer momento, esta teoría económica tuvo un correlato político, relacionado no tanto con la necesidad de mantener incontaminados los mecanismos mercantiles como con los subproductos políticos positivos de su extensión social (Hirschman, 1977). Se trata de una doctrina que se remonta al menos a Montesquieu y Bentham y llega hasta Hayek y Friedmann, y plantea que la coordinación, a través de mecanismos de mercado del mayor número posible de áreas sociales, es la única garantía de preservación de la libertad individual en sociedades de masas y culturalmente plurales en las que las posibilidades alcanzar la unanimidad, a través de la deliberación, son muy reducidas y, por lo tanto, siempre está presente la amenaza del conflicto abierto o la imposición de las mayorías sobre las minorías.

Debemos a Karl Polanyi (2015) el haber sacado a la luz con mucha claridad que ambas tesis acerca de los efectos de la mercantilización adolecen de importantes sesgos ideológicos. En primer lugar, el mercado libre nunca ha existido. Los procesos históricos de mercantilización siempre han estado acompañados de intensas - y generalmente violentas e impositivas - intervenciones del Estado dirigidas a proteger los intereses de las clases dominantes de sus fallos sistémicos y sus externalidades negativas. Se trata de una ortopedia política ideológicamente oculta, no sometida a escrutinio público ni a deliberación democrática, en la medida en que se presenta como una corrección puntual que remedia algún problema concreto del sistema de coordinación autorregulada, y no como su estructura propiamente dicha. En segundo lugar, como ya - desde finales del siglo XIXseñalaron los teóricos del imperialismo, la intervención estatal ha sido crucial para impulsar los procesos de mercantilización y quebrar la resistencia popular a aceptar la proletarización. La tesis de que el comercio suaviza los conflictos colectivos opaca la re- 
presión y los procesos de resistencia que ha generado la subordinación global de la sociedad al ideal del mercado libre generalizado.

De ahí la paradoja de que la extensión de la mercantilización no produjera una reducción del control y la coordinación estatal sino que, muy al contrario, fue contemporánea del desarrollo de mecanismos burocráticos de control social a una escala nunca conocida. Desde el siglo XVIII hasta hoy, la era del mercado es también la época del panóptico. A su vez, como han señalaron historiadores como Edward Palmer Thompson (1977) o Eric Hobsbawm (1979), las clases populares no se limitaron a proponer estrategias reaccionarias arcaizantes, sino que trataron de introducir fricción social en los procesos de proletarización, desarrollando instituciones de apoyo mutuo novedosas que, en ocasiones, tenían un fuerte componente ilustrado y emancipador. Esta dialéctica entre las estrategias de intervención estatal crecientemente sofisticadas - y con muy distintas modulaciones éticas y políticas - y las resistencias populares basadas en la solidaridad entre iguales marcó decisivamente la aparición de instrumentos públicos de embridamiento del libre mercado o de contención de sus efectos más perniciosos que, al mismo tiempo, podían tener dimensiones disciplinarias cruciales, una tensión muy presente en el desarrollo del Estado de bienestar tras la Segunda Guerra Mundial. Ese es precisamente el objeto de una importante familia de estudios de inspiración foucaultiana - de Robert Castel a Loïc Wacquant pasando por Jacques Donzelotcentrados en el análisis de las instituciones estatales de contención de la conflictividad más allá de la temática weberiana de la racionalización.

\section{El Trabajo Social como modo específi- co de gobierno de lo social}

El background del Trabajo Social está atravesado por un relato histórico que se relaciona de una manera ambigua con los precedentes benéfico-caritativos de la profesión: por un lado, reconociendo su herencia y, por el otro, afirmando una ruptura respecto a la represión de la pobreza que encarnaban. El postulado del surgimiento de una profesión de ayuda - antes que de control - y preventiva - antes que represiva - se levanta sobre el reconocimiento de figuras como la de Juan Luis Vives y su meritorio acoplamiento entre las exigencias capitalistas y la asistencia religiosa medieval (Castel, 1997), la diferenciación respecto de la policía del siglo XVIII (que se encargaba en las ciudades francesas de la salud de la población, la educación de los niños, el comercio y la reducción de vagabundos mediante la obligación de trabajar) (Castel, 1997; Foucault, 2008), o el rechazo a la Ley de Pobres inglesa de 1834 que, ante los elevados costes de la beneficencia reprimió la pobreza y alentó el empleo de técnicas refinadas de valoración (que distinguieran entre «pobres merecedores»y «no merecedores») y de intervención (sobre la educación de los hijos, condicionalidad de las ayudas y registro de los perceptores) (Donzelot, 1998). Las organizaciones caritativas que surgieron de esta deriva perseguían de forma obsesiva generar incomodidad en la pobreza como vía para la introducción de la ética del trabajo, la idea de autosuficiencia y la mejora moral.

Esta filantropía caritativa con aspiraciones científicas -e inspirada en el modelo médico-liberal (Álvarez-Uría, 1995)—constituye el precedente más inmediato del Trabajo Social, pero también es señalado habitualmente como el contraste negativo sobre el cual se construyó un modo alternativo de relacionarse con la pobreza que no pasaba por su culpabilización. Pese a la supervivencia histórica, que llega hasta nuestros días, de múltiples prácticas benéfico-caritativas que llevan a cabo instituciones eclesiásticas y organizaciones filantrópicas, una nueva perspectiva reformista - en el seno del progresismo liberal - alertada por el riesgo de fractura social ante las crecientes desigualdades, cuestionó a partir de finales del siglo XIX el darwinismo social («dejar caer a los débiles») que iba ganando hegemonía cultural. El resultado fue, como sabemos, la emergencia de la asistencia social como profesión con vocación de superar los modos de inter- 
vención propios de las organizaciones caritativas (centrados en la responsabilidad individual y los vicios morales). Un buen ejemplo lo constituyen los centros sociales que promovió Jane Addams, que eran definidos como «un esfuerzo experimental para ayudar a la solución de los problemas sociales e industriales que son generados por las condiciones de la vida moderna en una gran ciudad» (2013, p. 86). A través de programas educativos experimentales, de la cesión de espacios para la auto-organización sindical, de la práctica del mestizaje social entre sujetos de distinta condición (inmigrantes pobres y universitarios acomodados), de una visión positiva de las personas que usaban los settlements (en lugar de desconfianza a su parasitismo) y del impulso de reformas sociales que atenuasen la desigualdad, se estaba produciendo una ruptura respecto a la visión caritativa, des-culpabilizando la pobreza y relacionando las prácticas de los pobres con un contexto de falta de oportunidades (AlvárezUría y Parra, 2014).

No obstante, el alcance de esta ruptura era limitado en relación a las prácticas instituyentes que se venían practicando de forma autónoma en el movimiento obrero, y especialmente a partir de la Comuna de París y las ideas de Pierre-Joseph Proudhon (Laval y Dardot, 2015). El cuestionamiento de las estructuras de propiedad privada sacralizadas por el liberalismo y la construcción de relaciones comunales de apoyo mutuo (sindicatos, cooperativas y mutuas), constituían formas de autoorganización obrera que neutralizaban la individualización y la competencia entre las clases populares sin producir instituciones, profesionales y saberes ajenos a las mismas.

Por otro lado, al tiempo que se legitimaban los discursos y prácticas progresistas seminales en el Trabajo Social, fueron perdiendo capacidad transformadora: las condiciones de posibilidad de su desarrollo tuvieron que ver con la inserción de la asistencia social en los dispositivos judiciales (y la posterior conformación de los servicios sociales bajo una lógica judicial) y con la traslación de la lógica filantrópico-asistencial a las instituciones estatales. Los principios comprensivos y edu- cativos con los que nació el Trabajo Social podían seguir trabajando en un nuevo contexto institucional, pero ahora con funciones de control específicas. Como destacó Jacques Donzelot para el caso francés, la especial vinculación de la asistencia social a los problemas de la infancia a principios del siglo $\mathrm{XX}$ puso de relieve los abordajes preventivos por contraposición a los represivos. Detrás de un niño delincuente había una historia familiar que había que indagar para predecir su comportamiento e introducir medidas educativas, especialmente vehiculadas por la madre. Asistimos a un «paradójico resultado de la liberalización de la familia, del nacimiento de un derecho del niño, del reajuste de la relación hombre-mujer: cuanto más son proclamados estos derechos, más se estrecha al rededor de la familia pobre el cerco de un poder tutelar» (Donzelot, 1998, p. 106). La búsqueda de la alianza con la madre como sujeto socializador en el interior de la familia por parte de los técnicos al servicio de la institución tutelar sustituía el patriarcado familiar por el patriarcado institucional. Desde lo blando-femenino de las instituciones se desarrolla una intervención capilar en la vida cotidiana de las clases populares (Grassi, 1989), y lo que nace como una reacción a la represión de los pobres y los delincuentes en la era liberal, el concepto de prevención y su ligazón con una visión educativa, acaba funcionando como la habilitación de una serie de prácticas sociocéntricas de control.

La extensión de sistemas de protección social, a partir de las propuestas keynesianas (seguros de desempleo, pensiones, sanidad, etc.), reubicó al Trabajo Social: por un lado, en la gestión de prestaciones asistenciales por fuera de la cobertura de los sistemas universales y, por el otro, en la intervención relacional sobre los problemas sociales encarnados por las poblaciones populares. En Estados Unidos, tras la decadencia de los settlements, se fortaleció un enfoque individual bajo la influencia del psicoanálisis, al tiempo que iban ganando peso conceptos centrados en la gestión racional de recursos de arriba abajo, como el de cambio planificado (organización de la ayuda a partir del conocimiento de la 
comunidad destinataria para evitar duplicidades y perseguir la eficacia). En Europa, el incremento de los salarios directos e indirectos, por medio de la implantación de políticas sociales redistributivas y universalistas, convirtió en objeto preferente de la intervención familiar tanto a los sujetos pobres y marginales que quedaban fuera de las protecciones al salariado, como a las comunidades rurales y urbanas «subdesarrolladas» con respecto al modelo industrial (a las que se aplicaban las técnicas del desarrollo comunitario, el mismo que Naciones Unidas venía introduciendo en los países descolonizados). Sin embargo, los límites integradores del «welfarismo» acabarían constituyendo la base para la estigmatización de las poblaciones objeto del Trabajo Social, las cuales no estarían «aprovechando las oportunidades» que se les brindaban (Sales, 2014). Se abría así la puerta a una nueva ronda de discursos centrados en la responsabilidad individual de la pobreza.

\section{Críticas al Trabajo Social en tiempos «welfaristas»}

El rol asistencial y funcionalista del Trabajo Social fue objeto al menos de tres grupos de críticas radicales, a partir de las décadas de 1960 y 1970 . La primera de ellas planteaba en Estados Unidos la defensa de las comunidades excluidas de los derechos asociados al fordismo-welfarismo frente a los modelos adaptativos-consensuales de Trabajo Social. Desde la perspectiva de una práctica conflictiva de la organización comunitaria, se cuestionaban de forma pragmática los poderes locales establecidos (empresas, instituciones públicas, etc.), tratando de arrancar mejoras sociales y laborales en los barrios deprimidos de las ciudades norteamericanas, y de eludir la represión a través de tácticas imaginativas desde el interior del marco de experiencia de dichas comunidades (Alinsky, 2013). A su vez, la organización de los Panteras Negras desarrolló sistemas comunitarios de apoyo mutuo y autogestión en los guetos de las grandes ciudades norteamericanas, y el Trabajo
Social de defensa y potenciación de las comunidades negras oprimidas abriría la puerta a la extensión del concepto de empoderamiento (Solomon, 1976; Pastor, 2015). Dicho concepto, entendido como desarrollo del sentido de pertenencia e identidad en los colectivos «inferiorizados» (no solo negros, sino también mujeres, indígenas, etc.) a partir del análisis causal y estructural del propio sufrimiento social, fue también adoptado por los grupos de autoconciencia feminista (Dominelli y MacLeod, 1999).

El segundo grupo de críticas al Trabajo Social adaptativo propio de la era welfaristaasistencial destacó su funcionalidad como modo de alienación. Entre las décadas de 1960 y 1970, en un contexto de empuje de los movimientos populares-nacionales en Latinoamérica, proliferaron los discursos de inspiración marxista de la reconceptualización en el Trabajo Social, acompañados por la emergencia de las teorías de la dependencia, la teología de la liberación, las pedagogías «concientizadoras», la psicología comunitaria y el teatro social. En dicho contexto, al Trabajo Social se le atribuiría una posición de alianza con las clases populares y una misión «concienciatizadora» de base comunitaria frente al trabajo funcionalista de corte individual (Alayón, 2005). Al margen de algunos desarrollos teóricos que tuvieron un inmenso calado en la conformación de la identidad profesional, tanto en Latinoamérica como en España, algunas traducciones prácticas de estas críticas se asocian al desarrollo de diversas metodologías participativas entre sectores rurales y urbanos periféricos como modo de producir sujetos activos de cambio en la remoción de las estructuras sociales. Hay que destacar, además, la importación del enfoque reconceptualizador por parte del movimiento de cristianos de base en la España de la Transición, el cual animó junto con el movimiento vecinal un trabajo comunitario de alianza con las periferias urbanas en la reivindicación de mejoras sociales y del llamado derecho a la ciudad.

El tercer y último conjunto de críticas hay que situarlo geográficamente sobre todo en Europa y se centró en denunciar el carácter 
violentamente disciplinario de las instituciones. Los vientos contraculturales que soplaron en Mayo de 1968 encontraron en las investigaciones sobre las instituciones totales de Erving Goffman (1972), en la genealogía sobre el nacimiento de la disciplina por parte de Michel Foucault (2002), en el movimiento de la antipsiquiatría de Franco Basaglia (2005) y en el análisis institucional de René Lourau (2000), el aire fresco que vendría a hacer visibles las relaciones de poder en la intervención experta a través de la producción de la subjetividad (del enfermo, del preso o del asistido). La principal contribución de este grupo de análisis críticos fue el impulso de la democratización en el interior de la relación profesional e incluso la desinstitucionalización y desprofesionalización de ciertas prácticas de apoyo mutuo a través de un reordenamiento menos jerarquizado de los roles (Vercauteren, Crabbe, y Müller, 2010; Rendueles, 2010; Barrilete Cósmico, 2011).

Como puede apreciarse, estos tres grupos de críticas al - y en el- Trabajo Social no pueden entenderse sin los movimientos políticos y sociales que en cada contexto sociohistórico contribuyeron a deconstruir los relatos hegemónicos. Como afirman Michael Lavalette e Iain Ferguson (Lavalette, 2014), los cambios experimentados por la disciplina no pueden entenderse de forma autónoma sino acudiendo precisamente a dichos movimientos. La estrategia de lectura que proponemos consiste en prestar atención precisamente a la incorporación exitosa de esas críticas en la intervención social con el fin de captar las claves de gobierno de lo social que en el neoliberalismo se derivarán de ellas.

\section{La contrarreforma neoliberal y la «fra- gilización» social}

El ascenso del neoliberalismo fue el resultado de un proceso relativamente rápido de ofensiva de las clases altas tras la oleada insurreccional de 1968 y el inicio de la crisis económica de 1973. En cierto sentido, puede entenderse como un proceso de restauración del capitalismo manchesteriano tras el paréntesis keynesiano de la postguerrra, un entorno social en el que la libre empresa carece de limitaciones y está respaldada por instituciones dirigidas a proteger la propiedad privada y la libertad comercial. Y, al igual que en el pasado, el proyecto de mercantilización neoliberal muestra una clara ambivalencia entre, por un lado, el rechazo ideológico de la intervención del Estado y, por otro, el recurso sistemático a herramientas represivas y burocráticas de control, gestión y contención social (Birch y Mykhnenko, 2010; Manfred y Roy, 2011; Dumenil, 2014).

Lo cierto es que el núcleo vertebrador de las políticas neoliberales ha sido el desarrollo de un modelo de acumulación capaz de superar la crisis global que atravesaba el sistema capitalista sin cuestionar la posición de privilegio de las élites económicas. Aunque la exaltación de la libertad y la creatividad individual han sido elementos discursivos esenciales en la construcción de una ideología eficaz, su materialización política ha estado supeditada en todo momento a la preservación de los beneficios capitalistas y en ningún momento ha sido incompatible, más bien al contrario, con las dictaduras militares, el incremento del gasto público armamentístico o penitenciario o la socialización de las pérdidas privadas a través de rescates bancarios. De hecho, la «financiarización» contemporánea de la economía es mucho más compleja que la tradicional precisamente porque las crisis generadas por la propia economía especulativa han sido utilizadas como ventanas de oportunidad para aplicar estrategias de shock privatizadoras (Harvey, 2007).

En cualquier caso, el éxito de esta estrategia en términos de dominio de clase es incuestionable. Desde principios de los años noventa, las políticas neoliberales redefinieron un sentido común global, estableciendo nuevos límites de lo que se consideraba socialmente posible, imposible e inaceptable. Fueron el fundamento de una nueva hegemonía: las clases capitalistas lograron asumir una función dirigente e interpelar a amplios sectores de las clases populares, que sintieron que sus intereses materiales se solapaban, al menos en parte, con los de las élites. Muy es- 
pecialmente, se produjo una derrota global del sindicalismo cuyo efecto más visible fue un brusco descenso de la participación de las clases trabajadoras en la riqueza nacional y un aumento de la desigualdad. Sin embargo, tan importante como la polarización económica, ha sido la «fragilización» social inducida por la mercantilización (Sennet, 2012; Therborn, 2015).

El fundamento de la desactivación de la lucha de clases desde abajo, característica del capitalismo contemporáneo, es la individualización generalizada que ha privado a los asalariados de una comunidad laboral solidaria y ha obligado a buscar estrategias personales de mejora del poder de negociación (en particular a través de la formación y del endeudamiento hipotecario como vía de movilidad social ascendente intergeneracional). Este proceso de desmembración ha generado un amplio conjunto de malestares y conflictos sociales que los gobiernos han tenido que atajar a través de nuevos instrumentos de contención: workfare, políticas penales punitivas, estrategias de flexibilización laboral... (Standing, 2013)

En este escenario, las respuestas contrahegemónicas contemporáneas no han sido capaces de ofrecer una alternativa mayoritaria. A menudo han oscilado entre el anhelo nostálgico de una restauración keynesiana y el pensamiento desiderativo, entendiendo la extensión de la mercantilización como la semilla de un inminente florecimiento histórico de posibilidades emancipatorias. En particular, el utopismo tecnológico ha concentrado gran parte de este optimismo inane, que ha querido ver en el entorno digital la matriz de nuevas formas cooperativas capaces de desafiar el orden neoliberal y en el nuevo panóptico digital la principal amenaza a esa dinámica antagonista. De este modo, se ha tendido a opacar en algunas de las reflexiones críticas de mayor impacto el papel crucial que siguen desempeñando los mecanismos de intervención social estatal tradicionales - regulación del mercado de trabajo, políticas educativas, nuevo régimen fiscal, crisis de los cuidados, gestión de las ayudas sociales, legislación penal... - y el modo en que se han ido transformando para adaptarse a las nuevas necesidades de contención del riesgo social.

\section{La intervención social en el neolibera- lismo}

Apenas existen análisis empíricos y reflexiones teóricas en España sobre la influencia del neoliberalismo en el Trabajo Social. El vocablo neoliberalismo suele asociarse a las políticas de austeridad impuestas durante la última crisis económica, pero esta lectura ignora que las intervenciones neoliberales se vienen produciendo desde hace tres décadas y sus efectos sociales trascienden el «austericidio». El neoliberalismo es también un modo de producir una sociedad asimilada cada vez más en su funcionamiento al mercado y un modo de gobierno basado en el estímulo de la competencia por medio de la desigualdad (Laval y Dardot, 2013). La extensión de la competencia entre sujetos, instituciones y colectivos traslada la lógica de la empresa a toda la sociedad (tanto a lo público como a los individuos, impelidos a convertirse en empresarios de sí) (Foucault, 2007), una forma de consolidar el homo æeconomicus y el interés privado como única subjetividad posible y deseable (régimen de verdad). Una sociedad formada por empresarios de sí mismos, eficientes calculadores de costes y beneficios que asumen riesgos en lugar de esperar que les proteja la comunidad o el Estado, extiende las tecnologías de control al interior de los propios sujetos: es la prevención respecto de los propios riesgos y respecto de los que encarnan los otros, lo que logra generar mecanismos de confianza en uno mismo - capital humano, empoderamiento individual, emprendimiento - y desconfianza respecto de los demás - comparación, inseguridad, rivalidad - . Desde esta perspectiva, a las políticas sociales no les compete reducir la desigualdad sino promover la responsabilidad individual y, cuando esto fracasa, orquestar los dispositivos necesarios para la detección, prevención y contención de riesgos sociales (la pobreza, la violencia y la protesta) (Ávila y García, 2013). En lo que sigue, mapea- 
remos algunas de las manifestaciones de la gubernamentalidad neoliberal de lo social divididas en dos apartados: uno referido a las condiciones materiales y organizacionales que impone y otro dedicado a las subjetividades que construye. Ambos recorridos los haremos acompañados de algunas de las críticas que ha recibido la intervención social neoliberal.

\subsection{Implicaciones materiales y organiza- cionales de la intervención social neoliberal}

Englobamos aquí las características más sobresalientes de los objetivos que organizan las políticas sociales y el Trabajo Social en el contexto contemporáneo.

\subsubsection{Austeridad}

La primera de las manifestaciones de la política social neoliberal es la austeridad. Aunque no es la única característica del neoliberalismo, sería clamoroso obviar su importancia. El cálculo de costes y beneficios desde parámetros mercantiles convierte ciertas políticas sociales en lastres para el crecimiento económico («déficit»). Así es como los recortes de presupuestos y plantilla en materia de servicios sociales contribuyen a equilibrar la balanza fiscal (en lugar del cese de la reducción de la carga fiscal sobre las clases altas). Desde el Trabajo Social radical británico se vienen denunciando estas políticas de austeridad y el ataque al potencial transformador que encarna el propio Trabajo Social, así como los efectos sobre los colectivos más vulnerables (mujeres, minorías, etc.) (Lavalette, 2014). En el sur de Europa, especialmente castigado por la disciplina de la austeridad, múltiples voces han venido denunciando el frenazo al incompleto desarrollo de los servicios sociales (Alguacil, 2012), la desinversión pública en materia de pobreza, dependencia, etc. (Martínez, Cruz y Ioakimidis, 2014; Asociación Estatal de Directores y Gerentes de Servicios Sociales, 2016), así como el neofilantropismo en forma de shows de humanitarismo y de bancos de alimentos (Nogués y Cabrera, 2017).
5.1.2. Igualdad de oportunidades, focalización y activación

Una segunda manifestación del neoliberalismo en la organización de lo social lo constituye la definición del objeto mismo de la política social. Los objetivos redistributivos de arriba abajo de las políticas sociales welfaristas resultan ahora perniciosos desde una perspectiva que no ve en la desigualdad un problema, sino un estímulo para la activación de quienes menos tienen, y por lo tanto para el progreso general de la sociedad. En el contexto español, las políticas sociales de los primeros gobiernos socialistas en la década de 1980 ya llevaban preñado el concepto de «igualdad de oportunidades», introduciendo el matiz de la necesaria competitividad en una sociedad de mercado sobre la vieja noción socialista de igualdad. El marco de verdad que se impuso en la Transición anticipó que la desigualdad sería «inevitable», pero a cambio el país se modernizaría con su entrada en la Comunidad Económica Europea trayendo desarrollo para todos (efecto ascensor) (Colectivo IOE, 1990).

En otras latitudes, también hemos asistido al declive del objetivo político de la igualdad. La focalización en la pobreza y la exclusión, y la especial atención a la formación para el empleo, la productividad de los pobres, su activación para el mercado, la innovación para el emprendimiento y el estímulo económico mediante microcréditos, han sido centrales en las políticas sociales de segunda generación en Latinoamérica durante las últimas décadas (Aquín, 1999; Coraggio, 2008). Raúl Zibechi ve en estas políticas el sello del Banco Mundial y los aprendizajes de su director, Robert McNamara, cuando fue Secretario de Defensa estadounidense: tras el fracaso de Estados Unidos en la Guerra de Vietnam, se hizo evidente que el conflicto bélico era insuficiente si no iba acompañado de la lucha contrainsurgente por medio de políticas sociales (Zibechi, 2012; Neocleous, 2016). Esta filosofía fue trasladada a Latinoamérica en las décadas de 1980 y 1990 por medio del trabajo con ONGs locales, pero Zibechi va aún más lejos en su crítica a estas 
políticas sociales, detectando precisamente su proyección, y no su cortocircuito, en el contexto de los gobiernos progresistas de la región en la década de 2000 (la Argentina de los Kirchner, el Brasil de Lula y Roussef, etc.). Esta segunda generación de políticas sociales ya no trabaja sobre la privatización y la ruptura del vínculo social, sino que trata de instrumentalizar el propio vínculo formado en los ciclos de protestas (cooperativas de trabajadores pobres o redes de trueque comunitarias surgidas en la crisis argentina de 2001) con el fin desactivarlo en su potencia política. Convertir a los movimientos en organizaciones, la autogestión en actividad profesional y a los militantes en técnicos, resultó eficaz a la hora de reducir la conflictividad social, al tiempo que las políticas económicas no tocaban las estructuras de propiedad y las actividades acumulativas y extractivistas (Zibechi, 2012).

Estas políticas sociales focalizadas en la pobreza - y no en la desigualdad - y en la activación para el mercado - y no en el autogobierno-, han caracterizado también las acciones públicas y del tercer sector en Europa, y específicamente en España. Desde que en la década de 1970 se introduce el concepto de exclusión y se acompaña de nuevas políticas sociales, fundamentalmente a través de los programas de rentas mínimas de inserción, se han ido sedimentando una serie de discursos y conceptos que van abandonando abordajes estructurales no centrados solo en la pobreza sino también en la riqueza. Como ya señaló Michel Foucault, a finales de la década de 1970, en el cuerpo doctrinal neoliberal no se elimina la política social, sino que se piensa fundamentalmente como un modo de aliviar a quienes han caído fuera del juego de la competencia para devolverlos a la misma: la invención de las rentas mínimas de inserción en Francia tenía este sentido, proporcionar un mínimo que no acomodase al perceptor a cambio de acciones para su activación y reinserción competitiva (Foucault, 2008). El foco sobre la pobreza y la exclusión a través de su monitoreo mediante la producción de datos, índices e indicadores, da cuenta de una gestión biopolítica inseparable del mercado. La Unión Europea a través de la Estrategia Europa 2020, la Administración del Estado mediante el Plan Nacional de Acción para la Inclusión Social del Reino de España 2013-2016, o la Comunidad de Madrid a través de la Estrategia de Inclusión Social de la Comunidad de Madrid 2016-2021, han ido asumiendo los principios de la «inclusión activa», basada en un «apoyo a la renta» de los más excluidos, el «acceso al empleo» como fundamental indicador de inserción y unos «servicios públicos de calidad» que promuevan la «inclusión social y económica» (Comunidad de Madrid, 2016). Los recursos destinados a la formación y el fomento del emprendimiento de los excluidos a través de microcréditos (Rodríguez, 2016), son algunas de las nuevas vetas que se exploran en estas estrategias de inclusión, asumiendo como único criterio de la misma la inserción en el mercado. Como no se cansan de repetir quienes abrazan los dogmas neoliberales, la mejor política social es la creación de empleo, pero cabría añadir como objetivo fundamental la producción de sujetos empleables (Font y Langarita, 2015).

\subsubsection{Gerencialismo}

Un tercer indicador de la impronta neoliberal en lo social nos lo proporcionan los cambios producidos en las instituciones que lo gestionan. Los servicios sociales han sido un campo especialmente afectado por las externalizaciones, privatizándose la gestión de servicios ya existentes o diseñando los nuevos bajo gestión privada. La creación de nuevos nichos de negocio para grandes empresas (en los cuidados, en la intervención socioeducativa, en la mediación, etc.), y el apremio para hacer funcionar bajo lógicas mercantiles a organizaciones del tercer sector y a pequeñas asociaciones de carácter local, introduce la lógica de la competencia en un campo en el que a priori podrían predominar formas cooperativas y de solidaridad. Pero además, constituye un eficaz modo de gobernar el potencial crítico de muchas organizaciones del tercer sector que dependen de los contratos y subvenciones. Por último, no hay que olvidar 
que como modo de gestión genuinamente neoliberal, mediante las externalizaciones el Estado no pierde capacidad de dirección política, sino que gobierna de manera indirecta y con un margen de flexibilidad mayor que las viejas estructuras estatales los riesgos sociales variables (Ávila y Malo, 2009).

No obstante, una suerte de corriente neofilantrópica va haciendo emerger como sujetos de la acción social a fundaciones con una amplia capacidad presupuestaria y también con un amplio margen de gobierno sobre los criterios de la intervención. La Responsabilidad Social Corporativa (RSC) constituye cada vez más una estrategia central en las empresas. Entre los beneficios para las mismas, se encuentra la proyección de una imagen de compromiso social que tape la lógica de beneficio privado, la generación de nuevos mercados, o la «obligación» de restituir a la sociedad lo que se ha extraído de ella, pero otorgando a esas mismas empresas, y no ya al Estado o a las comunidades, el poder de diseñar las acciones sociales. Como parte de la literatura sobre Responsabilidad Social Corporativa reconoce, si el entorno de la empresa se deteriora, la empresa se devaluará (Secchi, 2007). Los casos más llamativos son los de las fundaciones bancarias, las cuales desarrollan acciones asistenciales (cheques para familias pobres), comunitarias (proyectos de intervención comunitaria intercultural en barrios degradados) o de gestión de vivienda social (precisamente, el parque de vivienda que ha quedado vacío como consecuencia de los desahucios ejecutados por los mismos bancos).

Pero la lógica mercantil no consiste solo en la ampliación del campo de acción privada, sino también en la introducción de esa dinámica en el funcionamiento del Estado. La retórica gerencial ha ido penetrando en los servicios sociales del mismo modo que lo ha hecho en la policía, la educación o la sanidad. El énfasis en las habilidades de gestión, en las mediciones de rendimiento cuantificables y en la evaluación, se ha generalizado en las instituciones de Trabajo Social (Ferguson, 2012). Se ha impuesto una lógica económica (de análisis de costes y beneficios) y de auditoría continua que centra su atención en los datos más que en los contenidos (lo cual supone una re-burocratización en forma de informes, memorias, etc.) e introduce las estrategias del management empresarial en los servicios sociales en detrimento de las identidades profesionales y del saber hacer que pudieron enarbolar los trabajadores sociales en la transmisión del programa institucional en un contexto welfarista-disciplinario (Dubet, 2006). Se trata de una auténtica colonización por parte de la eficiencia economicista de la gestión de la pobreza (Grassi, 2003).

Si las instituciones se convierten cada vez más en empresas y los usuarios en clientes a los que se les da la posibilidad de elegir (Rose, 1997), no cabe extrañarse de la irrupción del marketing en forma de campañas publicitarias, cartas de servicios, sellos de calidad y llamadas al buen trato relacional del ciudadano (como modo de compensar el maltrato material y la ausencia de democracia en dichas instituciones). La «sonrisa de la institución» (Velasco, Díaz de Rada, Cruces, Fernández, Jiménez y Sánchez, 2010) contrasta, sin embargo, con una enorme burocratización que ya se manifestó en forma de malestar profesional en los primeros años de los servicios sociales (Álvarez-Uría, 1995), pero que hay que entender además en su funcionalidad actual en el marco de austeridad: la burodisuasión, la buroexclusión y la burorrepresión constituyen mecanismos despersonalizados y dispersos de regulación en el acceso a los derechos (Ávila, Domínguez, García, Maroto, Martín, Oliver, 2015).

\subsection{La subjetivación neoliberal a través de la intervención social}

Indisociable de las condiciones materiales y organizacionales que hemos descrito en el anterior apartado, hemos creído oportuno trabajar por separado los contenidos conceptuales que se ponen en boga en la intervención social contemporánea, a saber, la (neo)disciplina, el actuarialismo y la innovación social. Estos contenidos se realizan bien poniendo el acento en lo negativo - sujetando- o bien en lo positivo - produciendo. 
Entre las acciones en negativo destacamos la pervivencia de múltiples prácticas y gestos que consideran el Trabajo Social como un modo de disciplinamiento y castigo: el usuario es reducido a un conjunto de faltas y errores que deben ser reconducidas mediante intervenciones conductistas (combinando educación y sanción). El workfare asistencial acompañado de las políticas punitivas que describe Loïc Wacquant (2010) en EE UU tiene versiones más edulcoradas en nuestro contexto, si bien encuentra en el espíritu de algunos profesionales un campo para su desarrollo. Así es como, incluso contraviniendo las normas más garantistas, algunos profesionales usan la gestión de la renta mínima como un modo socio-céntrico de extender su propia cosmovisión moral (Ayala, 2009).

También en negativo, aunque no con una utopía moral de fondo sino buscando la eficiencia y la eficacia en el sostenimiento del orden urbano, buena parte de las energías de los servicios sociales y de proyectos del tercer sector están dedicadas a la gestión de riesgos y emergencias. Una policialización del Trabajo Social y una «trabajosocialización» de la policía se combinan para abordar de un modo actuarial la realidad de los barrios y las familias, llevando a cabo un continuo monitoreo, ejerciendo acciones preventivas banales y estableciendo protocolos de atención a las emergencias cuando no se han podido prevenir los riesgos. Este trabajo de parcheo tiene en los programas de poblaciones «en/de riesgo») (proyectos de prevención de bandas latinas, etc.) y en la atención a las emergencias sociales (Samur Social, etc.) sus desarrollos más singulares (Ávila y García, 2013).

Sin embargo, lo más genuino de la intervención neoliberal sobre lo social lo constituye la captura de conceptos que tuvieron un potencial crítico transformador y su amoldamiento al banal discurso del pensamiento positivo. Más arriba vimos cómo una serie de críticas en las décadas de 1960 y 1970 - Trabajo Social radical, reconceptualización, crítica anti-institucional - contribuyeron a erosionar por la izquierda el «welfarismo». Sin embargo, tras dejar su huella en la intervención social, estas tres críticas fueron perdien- do tanto su capacidad para comprender el nuevo contexto como su potencial transformador. Y es que, en buena medida, no fue tanto su derrota mediante la reacción de los poderes (aunque en Latinoamérica fueron muchos los trabajadores sociales represaliados por las dictaduras) como el éxito cultural de sus planteamientos, lo que constituyó la base para su desactivación política. Igual que el capitalismo aprende de las críticas recibidas (Boltanski y Chiapello, 2002) y ha sido capaz de ir incorporándolas a su funcionamiento - recontextualizadas y resignificadas - el Trabajo Social posible en el contexto neoliberal también ha ido bebiendo de los postulados que lo erosionaban, pero refuncionalizándolos de un modo tal que no hiciesen tambalearse las estructuras de poder capitalistas, coloniales y patriarcales. Se puede afirmar que los tres grupos de críticas dieron lugar a la centralidad de tres conceptos: del Trabajo Social radical surgió la idea de empoderamiento, la reconceptualización actualizó la participación y desde la crítica antiinstitucional se llegó a la innovación social. La razón de gobierno neoliberal ha ido redirigiendo el uso de dichos conceptos (que en función de los contextos pueden seguir albergando prácticas transformadoras) hacia la adaptación al mercado y la gobernanza del orden social desigual.

El empoderamiento, como proceso de toma de conciencia y capacitación para organizarse y movilizarse, atrae usos muy ambiguos y contradictorios entre sí (Bacque y Biewener, 2016). El concepto de empoderamiento ha sido en buena medida descolectivizado y psicologizado (de la dominación estructural a la «baja autoestima»), usado para adornar retóricamente intervenciones directivas y asistenciales («empoderar al otro») o para ser resignificado en la activación para el mercado sin cuestionar las estructuras dominantes («empoderamiento de las mujeres a través del emprendimiento»).

La participación, como evocación de la democracia directa ante los déficits de la democracia representativa, ha sido tenida en cuenta en las políticas públicas locales y en las acciones de las ONG, si bien sus usos ne- 
oliberales y socioliberales no siempre han ampliado la democracia, sino que la han encajonado en los marcos de la llamada «gobernanza». La participación ha sido instrumentalizada para ocuparse no de los beneficios que genera una sociedad sino de los costes (cuidados de personas dependientes a cargo de las familias y los vecindarios). Es el caso de la llamada «sociedad participativa» impulsada por el gobierno holandés para reducir la carga del Estado del bienestar (Subirats, 2013). También se usa la participación para delegar en las propias comunidades el control sobre los perceptores de las prestaciones (Rose, 1997; Agudo Sanchiz, 2009). Se dan otros usos retóricos de la participación o del trabajo comunitario donde solo hay charlas informativas y coordinación entre profesionales. La participación es más abierta en otros procesos barriales, pero quienes participan actúan como radares sociales que proporcionan información a las autoridades o como vecinos denunciantes, y no como sujetos reflexivos de deliberación y decisión. Se pueden observar procesos participativos en los que la población afectada decide, pero en el marco liberal de la elección racional, sin poder replantear las preguntas y sin poder impugnar las estructuras económicas y políticas. Por último, se cuestiona que la participación, aún en procesos muy abiertos, reflexivos y deliberativos, constituye un modo de arrancar a las comunidades de sus tejidos orgánicos - donde se proporcionan apoyo para la supervivencia y también «buena vida»_ para colocarlas en contextos formales «más pensados para ciudadanos abstractos que para tramas colectivas reales» (Izquierdo, 2017).

Por último, los cuestionamientos de las instituciones jerárquicas y la burocratización de la intervención social están dando lugar a la extensión de multitud de prácticas de innovación social basadas en las nuevas tecnologías y en las prácticas críticas de movimientos sociales para generar respuestas que ni la rigidez estatal ni la obsesión por la rentabilidad del mercado son capaces de abordar. Desde huertos urbanos a formas de economía colaborativa, pasando por aplicaciones informáticas de apoyo social y por prototipos ar- quitectónicos para mejorar un espacio comunitario, las acciones de innovación social están siendo cada vez más impulsadas por las instituciones públicas y el tercer sector. Sin embargo, en muchas ocasiones se confía a la innovación social la solución de problemas sociales sin pasar por la redistribución de renta y poder (Rendueles y Subirats, 2016; Castro y Martínez, 2016), aplicando recetas propias de las clases medias y las élites culturales a unos pobres que nuevamente mostrarán que no están a la altura de los tiempos (Martínez, 2014).

\section{Presentación de los artículos}

El hecho de que el neoliberalismo haya sido capaz de metabolizar los cuestionamientos a la intervención social, ¿implica necesariamente que la crítica esté condenada eternamente al fracaso? Los coordinadores de este número pensamos que el neoliberalismo plantea un nuevo campo de juego, más que el fin de la historia. Los efectos de los conceptos y las políticas sociales dependerán de su uso concreto en cada contexto. Las propuestas críticas que surgieron en las décadas de 1960 y 1970 poseen aún un amplio potencial crítico y, del mismo modo, aún perviven discursos y prácticas que en el siglo XIX sirvieron para denunciar la desigualdad capitalista y promover el apoyo mutuo. Además, la aparición contemporánea de nuevos conceptos y prácticas colectivas - comunes, cuidados, saberes subalternos... - puede ser el punto de partida de recorridos hoy insospechados para una comprensión emancipadora del Trabajo Social. De momento, quienes coordinamos este número solo pretendemos reabrir el campo de ideas y debates en torno a un Trabajo Social crítico.

Los artículos recogidos en este monográfico dan cuenta de la riqueza de las perspectivas críticas que hoy tratan de hacer frente a los desafíos del Trabajo Social. Así, uno de los signos de los tiempos en este campo de estudio es la recuperación y la actualización de los análisis biopolíticos de las estrategias de intervención social como mecanismos de 
contención y gestión del conflicto. La entrevista a Jacques Donzelot, uno de los pioneros en la aplicación de las metodologías genealógicas y arqueológicas al campo de la intervención social, presenta una evaluación retrospectiva de su propio trabajo en un momento de crisis de los instrumentos de solidaridad social característicos del Estado de bienestar. Lorenzo García Martín analiza las aproximaciones de Michel Foucault y el propio Jacques Donzelot a la intervención social como fuerza normalizadora que lleva los discursos higienistas, familiaristas y psicológicos hacia las clases populares. Por su parte, Manuel Desviat propone una evaluación crítica de la evolución de la reforma psiquiátrica y el Trabajo Social sanitario en un contexto de mercantilización generalizada. Las intervenciones psicosociales se han integrado en el sistema político-económico hegemónico y se ha desfigurado el espíritu original de la reforma - los principios salubristas, la desinstitucionalización real, el trabajo comunitario, la participación ciudadana...- en un proceso de patologización del sufrimiento social. Por último, María Inés Martínez Herrero mapea las mutaciones que ha sufrido en las últimas décadas el Trabajo Social en Inglaterra a la luz de su instrumentalización gubernamental para la introducción de la ideología neoliberal en el cuerpo social, así como las resistencias que surgen en el seno de un incipiente movimiento de renovación radical en la profesión. Se trata de un ejercicio de enorme trascendencia si tenemos en cuenta la influencia mundial que ha tenido y tiene dicho país en el nacimiento y desarrollo de la disciplina.

Un segundo conjunto de artículos se centra en los efectos de distintas herramientas concretas de intervención social en un contexto de aumento del sufrimiento social y la conflictividad. En primer lugar, dos artículos microsociológicos muestran la participación del Trabajo Social en las nuevas estrategias hegemónicas de contención del malestar. En concreto, Alejandro Agudo Sanchiz analiza el modo en que la dispersión de algunas funciones de gobernanza hacia el tercer sector no supone necesariamente un mayor protagonismo de la sociedad civil sino que a menu- do se trata de una transformación de las formas de intervención estatal que siguen siendo tan intensas como en el pasado. A través de un análisis etnográfico de un conjunto de intervenciones sociales en México saca a la luz la aparición de espacios ambiguos, donde la frontera entre lo público y lo privado se difumina, mientras la responsabilidad sobre los servicios sociales se descarga sobre los ciudadanos y las asociaciones civiles. Por su parte, Daniel Parajuá Navarrete hace una exploración de los dispositivos contemporáneos de codificación y monitorización de los usuarios de los servicios sociales por medio de las construcciones narrativas específicas de la intervención social. De este modo muestra la construcción institucional de los sujetos que son objeto de esa intervención, así como de algunas condiciones socio-históricas de producción de un tipo específico de asistencia social en el que existen tareas no explícitas relacionadas con el control de las poblaciones relegadas.

Otros dos textos de este bloque se centran en el análisis de las rentas mínimas como respuesta estatal a la crisis del pacto keynesiano-fordista y el fin del pleno empleo. En primer lugar, Joan Cortinas Muñoz estudia la historia institucional del proceso de implementación de la Renta Mínima de Inserción catalana, que fue diseñada como un dispositivo orientado a la capacitación profesional en proyectos de inserción locales a pesar de que el problema al que trata de dar respuesta tiene que ver con grandes mutaciones económicas y políticas globales. En segundo lugar, Rubén Lasheras Ruiz, Edurne Jabat Torres, Madalena D'oliveira-Martins e Ignacio Sánchez de la Yncera analizan el modo en que los perceptores de la Renta de Inclusión Social en Navarra tienden a asimilar una serie de dinámicas simbólicas que entran en conflicto con los propósitos inclusivos con los que fue diseñado el recurso: tendencia a la responsabilización individual por las situaciones de exclusión, exigencia de mayor supervisión por parte de los servicios sociales, sentimientos de vergüenza y culpa, etc.

El monográfico también presenta algunas experiencias de resistencia a la privatización 
y el tratamiento individualizador del malestar social. Enrique García Escamilla, David Muñoz Rodríguez y Manuel Sáez Bayona describen algunas de las claves teóricas y las estrategias de Baladre, un grupo español de coordinación de luchas contra el paro, el empobrecimiento y la exclusión social con más de treinta años de vida, que trata de actualizar concepciones críticas, liberadoras y emanci- padoras de Trabajo Social. Por su parte, Ivonne Herrera Pineda y Carlos Pereda Olarte exponen la labor de Invisibles de Tetuán, un colectivo que interviene en el distrito madrileño de Tetuán tratando de afrontar el desempleo, la precariedad laboral y la debilidad de los sistemas de protección social desde la auto-organización vecinal, el apoyo mutuo, el respeto a la diversidad y la metodología horizontal

\section{Referencias bibliográficas}

Addams, J (2013). Hull House: el valor de un centro social. Madrid: Consejo General del Trabajo Social-Paraninfo.

Agudo Sanchiz, A. (2009). Conocimiento, lenguaje, poder e intermediación. Perspectivas contemporáneas en la antropología de las políticas públicas. Estudios Sociológicos 27(79), 63-110. Disponible en: http://www.jstor.org/stable/25614134?seq=1\#page_scan_tab_contents

Alayón, N. (org.) (2005). Trabajo Social latinoamericano. A 40 años de la Reconceptualización. Buenos Aires: Espacio

Alguacil, J. (2012). La quiebra del incompleto sistema de Servicios Sociales en España. Cuadernos de Trabajo Social, 25(1), 63-74. Disponible en: http://revistas.ucm.es/index.php/CUTS/ article/view/38434/37175

Alinsky, S. (2013). Tratado para radicales. Manual para revolucionarios pragmáticos. Madrid: Traficantes de Sueños.

Álvarez-Uría, F. (1995). En torno a la crisis de los modelos de intervención social. En: VVAA, Desigualdad y pobreza hoy (pp. 5-39), Madrid: Talasa.

Álvárez-Uría, F. y Parra, P. (2014). The Bitter Cry: materiales para una genealogía de la identidad profesional de las pioneras del Trabajo Social en Inglaterra y los Estados Unidos. Cuadernos de Trabajo Social, 21(1), 93-102. Disponible en: http://revistas.ucm.es/index.php/CUTS/article/viewFile/ $43219 / 43070$

Aquín, N. (1999). Hacia la construcción de enfoques alternativos para el Trabajo Social para el nuevo milenio. Revista de Servicio Social, 3.Disponible en: www.catedras.fsoc.uba.ar/heler/aquin.doc

Asociación Estatal de Directores y Gerentes de Servicios Sociales. (2016). Evolución 2011-2016 de los datos básicos de gestión del sistema de atención a la dependencia y costes de financiación por comunidades autónomas. Recuperado de: http://www.directoressociales.com/images/documentos/ dictamenes/XVIdictamen/XVI\%20DICT\%20CCAA.pdf

Ávila, D., Domínguez, A., García, S. Maroto, M., Martín, Ó.J. y Oliver, P. (2015). La burorrepresión de la protesta y de la pobreza. En: D. Bondía (dir.), Defender a quien defiende. Leyes Mordaza y criminalización de la protesta en el Estado español (pp. 141-168). Barcelona: Icaria.

Ávila, D. y García, S. (2013). Entre el riesgo y la emergencia: insinuaciones policiales en la intervención social. Revista de Antropología Social, 22,59-82. Disponible en: http://revistas.ucm.es/ index.php/RASO/article/viewFile/43184/40946

Ávila, D. y Malo, M. (2009). Manos Invisibles. De la lógica neoliberal en lo social. Recuperado de http://manosinvisibles.net/wp-content/uploads/2013/09/manos_invisibles.pdf

Ayala, A. (2009). Secretos a voces: exclusión social y estrategias profesionales de construcción de la obligatoriedad a la Renta Mínima de Inserción (RMI) con el colectivo de etnia gitana. Cuadernos de Trabajo Social, 22, 19-40. Disponible en: http://revistas.ucm.es/index.php/CUTS/article/view/ CUTS0909110019A/7499 
Bacqué, M.H. y Biewener, C. (2016). El empoderamiento. Una acción progresiva que ha revolucionado la política y la sociedad. Barcelona: Gedisa.

Barrilete Cósmico (2011). Pura suerte. Pedagogía mutante. Territorio, encuentro y tiempo desquiciado. Buenos Aires: Tinta Limón.

Basaglia, F. (2005). L'utopia della realtà. Torino: Einaudi.

Birch, K. y Mykhnenko, V. (2010). The rise and fall of neoliberalism: the collapse of an economic order? Londres: Zed Books.

Boltanski, L. y Chiapello, E. (2002).El nuevo espíritu del capitalismo. Madrid: Akal.

Castel, R. (1997). La metamorfosis de la cuestión social. Una crónica del salariado. Buenos Aires: Editorial Paidós.

Castro, M. y Martínez, R. (2016). No hay participación sin redistribución del poder. Diagonal 29/04/16. Disponible en: https://www.diagonalperiodico.net/movimientos/30226-no-hay-participacion-sinredistribucion-del-poder.html

Colectivo IOE. (1990). Ideologías de la intervención social en la España de los 90. Documentación Social, 81, 53-64.

Comunidad de Madrid. (2016). Estrategia de Inclusión Social de la Comunidad de Madrid 2016-2021. Madrid: Dirección General de Servicios Sociales e Integración Social. Recuperado de: http:/www.madrid.org/cs/Satellite?blobcol=urldata\&blobheader=application\%2Fpdf\&blobheadername $1=$ Content - Disposition \&blobheadervalue $1=$ filename $\% 3$ DEstrategia + de + Inclusi\%C3\%B3n+Social+CM.pdf\&blobkey=id\&blobtable=MungoBlobs\&blobwhe$\mathrm{re}=1352918600937 \&$ ssbinary $=$ true

Coraggio, J.L. (2008). Crítica de la política social neoliberal: las nuevas tendencias. En: J. Ponce, Es posible pensar una nueva política social para América Latina, (pp. 81-94). Quito: FLACSO.

Dominelli, L. y MacLeod, E. (1999). Trabajo social feminista. Madrid: Cátedra.

Donzelot, J. (1998). La policía de las familias. Valencia: Pre-Textos.

Donzelot, J. (2007). La invención de lo social. Ensayos sobre la declinación de las pasiones políticas. Buenos Aires: Nueva Visión.

Dubet, F. (2006). El declive de la institución. Barcelona: Gedisa.

Dumenil, G. (2014). La crisis del neoliberalismo. Madrid: Lengua de Trapo

Ferguson, I. (2012). From Modernisation to Big Socitey: Continuity and Change in Social Work in the United Kingdom. Cuadernos de Trabajo Social, 25(1), 19-31. Disponible en: file://C:/Users/Q/ Downloads/38431-44972-2-PB.pdf

Font, J. y Langarita, J.A. (2015). A propósito de la inserción laboral. Implicaciones de las nuevas políticas de empleo para el Trabajo Social. Cuadernos de Trabajo Social, 28 (1), 61-68. Disponible en: file:///C:/Users/Q/Downloads/45253-85771-4-PB.pdf

Foucault, M. (2002). Vigilar y castigar. Nacimiento de la prisión. Buenos Aires: Siglo XXI.

Foucault, M. (2007). El nacimiento de la biopolítica. Buenos Aires: Fondo de Cultura Económica.

Foucault, M. (2008). Seguridad, territorio, población. Curso del Collège de France, 1976-1977. Madrid: Akal.

Goffman, E. (1972). Internados. Ensayos sobre la situación social de los enfermos mentales. Buenos Aires: Amorrortu.

Grassi, E. (1989). La mujer y la profesión de asistente social. El control de la vida cotidiana. Buenos Aires: Humanitas.

Grassi, E. (2003). Politicas y problemas sociales en la sociedad neoliberal. Buenos Aires: Espacio

Harvey, D. (2007). Breve historia del neoliberalismo. Madrid: Akal.

Hernández, D. (2012). Antes de empezar con metodologías participativas. Cuadernos CIMAS. Recuperado de: http://www.redcimas.org/wordpress/wp-content/uploads/2012/08/m_DHernandez_A NTES.pdf

Hirschman, A.O. (1977). Las pasiones y los intereses. Argumentos políticos en favor del capitalismo previos a su triunfo. Barcelona: Península. 
Hobsbawm, E.J.(1979). Trabajadores. Estudios de historia de la clase obrera. Barcelona: Crítica.

Izquierdo, A. (2017). La aurora de las tribus: entrevista a Andrés Izquierdo sobre política y socialidad en España. Recuperado de: http://anarquiacoronada.blogspot.com.es/2017/02/la-aurora-delas-tribus-entrevista.html

Laval, C. y Dardot, P. (2013). La nueva razón del mundo. Ensayo sobre la sociedad neoliberal. Barcelona: Editorial Gedisa.

Laval, C. y Dardot, P. (2015). Común. Ensayo sobre la revolución del siglo XXI. Madrid: Gedisa.

Lavalette, M. (2014). Entrevista al Profesor Michael Lavalette (Carmen Verde y Ana Lima). Servicios Sociales y Politica Social, 106, 161-169. Disponible en: http://www.serviciossocialesypoliticasocial.com/entrevista-al-profesor-michael-lavalette

Lourau, R. (2000). Libertad de movimientos. Una introducción al Análisis Institucional. Buenos Aires: Eudeba.

Martínez, R. (2014). La innovación social es de clase media. Nativa.cat 12/10/2014. Disponible en: http://www.nativa.cat/2014/10/la-innovacion-social-es-de-clase-media/

Martínez, I. Cruz, C. e Ioakimidis, V. (2014). Reconceptualizando el trabajo social en Europa del sur: El retorno de la política «en tiempos de malestares». Servicios Sociales y Política Social,106,1124. Disponible en: file:///C:/Users/Q/Downloads/1.-Reconceptualizando-el-trabajo-social-en-Europa-del-sur.-El-retorno-de-la-politica-en-tiempos-de-malestares\%20(1).pdf

Neocleous, M. (2016). La lógica de la pacificación: Guerra-Policía-Acumulación. Athenea Digital 16 (1), 9-22. Disponible en: file://C:/Users/Q/Downloads/1738-6633-2-PB.pdf

Nogués, L. y Cabrera, P. (2017). En España hay hambre: el derecho a la alimentación. Cuadernos de Trabajo Social, 30(1),11-16. Disponible en: file:///C:/Users/Q/Downloads/54646-105313-3-PB.pdf

Pastor, E. (2015). Trabajo Social con Comunidades. Madrid: Humanitas.

Perelman, M. (2000). The Invention of Capitalism. Londres: Duke University Press.

Polanyi, K. (2015). Los límites del mercado. Madrid: Capitán Swing.

Rendueles, C. y Subirats, J. (2016). Los bienes comunes, ¿Oportunidad o espejismo? Barcelona: Icaria.

Rendueles, G. (2010). Sólo una forma de vida en lo común permiten escapar a las miserias del individualismo o disminuir las penas cuando la tragedia nos alcanza (entrevista realizada por Salvador López Arnal). Rebelión. Disponible en: http://www.rebelion.org/noticia.php?id=87887

Rodríguez, J.R. (2016). Entreteniendo a los pobres. Una crítica político ideológica de las medidas de lucha contra la exclusión social. Albacete: Bomarzo.

Rose, N. (1997). El gobierno en las democracias liberales «avanzadas»: del liberalismo al neoliberalismo. Archipiélago, 29, 25-40.

Sales, A. (2014). El delito de ser pobre. Una gestión neoliberal de la marginalidad. Barcelona: Icaria.

Sennet, R. (2012). Juntos: rituales, placeres y políticas de cooperación. Barcelona: Anagrama.

Secchi, D. (2007). Utilitarian, managerial and relational theories of corporate social responsibility. International Journal of Management Reviews, 9(4),347-73. DOI: 10.1111/j.1468-2370.2007.00215.x

Solomon, B.B. (1976). Black Empowerment: Social Work in Oppressed Communities. Nueva York: Columbia University Press.

Standing, G. (2013). El precariado. Barcelona: Pasado y Presente.

Steger, M. y Roy, R. (2011). Neoliberalismo: una introducción. Madrid: Alianza.

Subirats, J. (2013). ¿Del Estado de bienestar a la sociedad participativa? El País, 28/09/2013, Disponible en: http:/ccaa.elpais.com/ccaa/2013/09/28/catalunya/1380395805_471576.html

Therborn, G. (2015). La desigualdad mata. Madrid: Alianza.

Thompson, E.P. (1963). La formación histórica de la clase obrera. Barcelona: Laia.

Velasco, H., Díaz de Rada, Á., Cruces, F., Fernández, R, Jímenez, C. y Sánchez, R. (2010). La sonrisa de la Institución. Confianza y riesgo en los sistemas expertos. Madrid: Editorial Centro de Estudios Ramón Areces.

Vercauteren, D., Crabbe, M. y Müller, T. (2010). Micropolíticas de los grupos. Para una ecología de las prácticas colectivas. Madrid: Traficantes de Sueños. 
Wacquant, L. (2010). Castigar a los pobres. El gobierno neoliberal de la inseguridad social. Barcelona: Gedisa.

Zibechi, R (2012). Política y miseria. Málaga: Zambra/Baladre. 